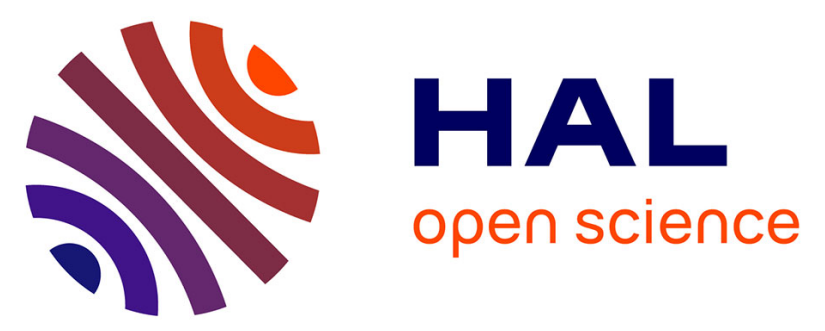

\title{
Bestimmung des Wirkstoffes Fenoxycarb in Holz mittels Hochdruckflüssigchromatographie
}

\author{
Ute Schoknecht, Claudia Bornkessel, Christina Fürhapper, Jan Gunschera, \\ Helmut Härtner, Rainer Hill, Jan Kochan, Eckhard Melcher, Ursula Wilken, \\ Joachim Wittenzellner, et al.
}

\section{To cite this version:}

Ute Schoknecht, Claudia Bornkessel, Christina Fürhapper, Jan Gunschera, Helmut Härtner, et al.. Bestimmung des Wirkstoffes Fenoxycarb in Holz mittels Hochdruckflüssigchromatographie. European Journal of Wood and Wood Products, 2009, 68 (4), pp.469-474. 10.1007/s00107-009-0387-0 . hal00568261

\section{HAL Id: hal-00568261 \\ https://hal.science/hal-00568261}

Submitted on 23 Feb 2011

HAL is a multi-disciplinary open access archive for the deposit and dissemination of scientific research documents, whether they are published or not. The documents may come from teaching and research institutions in France or abroad, or from public or private research centers.
L'archive ouverte pluridisciplinaire HAL, est destinée au dépôt et à la diffusion de documents scientifiques de niveau recherche, publiés ou non, émanant des établissements d'enseignement et de recherche français ou étrangers, des laboratoires publics ou privés. 


\section{焦 Springer}

Draft Manuscript for Review

\section{Bestimmung von Fenoxycarb in Holz mittels Hochdruckflüssigchromatographie}

\begin{tabular}{|r|l|}
\hline Journal: & Holz als Roh- und Werkstoff \\
\hline Manuscript ID: & HRW-09-0014.R1 \\
\hline Manuscript Type: & ORIGINALARBEITEN / ORIGINALS \\
\hline Author: & 29-May-2009 \\
\hline & $\begin{array}{l}\text { Complete List of Authors: } \\
\text { Fournkapsel, Claudia; Materialprüfungsanstalt Eberswalde GmbH } \\
\text { Gunschera, Jan; Fraunhofer-Institut für Holzforschung } \\
\text { Härtner, Helmut; RÜTGERS Organics GmbH } \\
\text { Hill, Rainer; Staatliche Materialprüfungsanstalt Darmstadt } \\
\text { Kochan, Jan; Janssen PMP } \\
\text { Melcher, Eckhard; vTI Institut für Holztechnologie und Holzbiologie } \\
\text { Wilken, Ursula; Remmers Baustofftechnik GmbH } \\
\text { Wittenzellner, Joachim; Dr. Wolman GmbH } \\
\text { Wobst, Matthias; Materialprüfanstalt für das Bauwesen }\end{array}$ \\
\hline Keywords: & $\begin{array}{l}\text { Biozid, Holzschutzmittel, Fenoxycarb, Holz, Analyse, } \\
\text { Hochdruckflüssigchromatographie }\end{array}$ \\
\hline
\end{tabular}

\section{$\$$ ScholarONE" \\ Manuscript Central}




\section{Bestimmung des Wirkstoffes von Fenoxycarb in Holz mittels Hochdruckflüssigchromatographie}

Schoknecht $\mathrm{U}^{1}$, Bornkessel, $\mathrm{C}^{2}$, Fürhapper $\mathrm{Ch}^{3}$, Gunschera $\mathrm{J}^{4}$, Härtner $\mathrm{H}^{5}$, Hill $\mathrm{R}^{6}$, Kochan, J. Melcher $\mathrm{E}^{8}$, Wilken $\mathrm{U}^{9}$, Wittenzellner $\mathrm{J}^{10}$, Wobst $\mathrm{M}^{11}$

1 Bundesanstalt für Materialforschung u. -prüfung, 12205 Berlin, Unter den Eichen 87

2 Materialprüfungsamt Eberswalde, 16225 Eberswalde, Alfred-Möller-Straße 1

3 Holzforschung Austria, A-1031 Wien, Franz-Grill-Straße 7

$4 \quad$ Fraunhofer Wilhelm-Klauditz-Institut, 38108 Braunschweig, Bienroder Weg 54 E

5 Rütgers Organics $\mathrm{GmbH}, 68305$ Mannheim, Sandhofer Straße 96

6 Staatliche Materialprüfungsanstalt Darmstadt, TU Darmstadt, 64283 Darmstadt, Grafenstraße 2

7 Janssen Preservation and Material Protection, B-2340 Beerse, Turnhoutseweg 30

8 Johann Heinrich von Thünen-Institut, 21031 Hamburg, Leuschnerstraße 91

9 Remmers Baustofftechnik GmbH, 49624 Löningen, Bernhard-Remmers-Straße 13

10 Dr. Wolman GmbH, 76547 Sinzheim, Dr.-Wolman-Straße 31-33

11 Materialprüfanstalt für das Bauwesen, TU Braunschweig, 38106 Braunschweig, Beethovenstraße 52

E-mail corresponding author: ute.schoknecht@bam.de

\section{Zusammenfassung}

Fenoxycarb ist ein insektizider Wirkstoff, der wegen seiner hormonähnlichen Wirkung in sehr geringen Konzentrationen in Holzschutzmitteln eingesetzt werden kann. Das hat zur Folge, dass aus Extrakten von behandeltem Holz relativ geringe Fenoxycarb-Mengen vor dem Hintergrund ebenfalls extrahierter Holzinhaltsstoffe zu bestimmen sind. In Vergleichsanalysen verschiedener Laboratorien wurde gezeigt, dass Fenoxycarb durch Methanol mit hoher Ausbeute aus Holz extrahierbar ist und in den Extrakten durch Hochdruckflüssigchromatographie in Kopplung mit UV-Detektion bestimmt werden kann. Fenoxycarb-Konzentrationen im unteren Bereich der erforderlichen Einbringmengen können mit dem erprobten Verfahren nur nach zusätzlicher Probenaufarbeitung bestimmt werden. Bedingt durch schwer auswertbare Chromatogramme sind die Abweichungen der Ergebnisse zwischen verschiedenen Laboratorien relativ hoch.

\section{Determination of Fenoxycarb in Timber using High Performance Liquid Chromatography}

Abstract: Fenoxycarb is an insecticide that can be used at very low concentrations in wood preservatives because of its hormon-like activity. As a consequence relatively low concentrations of fenoxycarb have to be determined in extracts of treated timber containing a background of wood extractives. It was demonstrated in parallel analyses in different laboratories that fenoxycarb can efficiently be extracted from treated wood by methanol and can be determined 
in the extracts by high performance liquid chromatography coupled with UV detection. However, fenoxycarb concentrations at the lower level of required retentions can only be determined according to the tested procedure with further sample preparation. The deviation of the results between the laboratories is relatively large caused by difficulties in the interpretation of the chromatograms.

\section{$1 \quad$ Einleitung}

Fenoxycarb (Ethyl[2-(4-phenoxyphenoxy)ethyl]carbaminsäureethylester, Strukturformel siehe Abbildung 1) wurde bei der Firma Maag Ltd. in der Schweiz entwickelt und durch Dorn et al. (1981) als nicht-neurotoxisches Insektizid mit juvenilhormonartiger Wirkung beschrieben. Durch die Firma Maag LTD (später Ciba-Geigy) wurde die Verbindung als Pflanzenschutzmittelwirkstoff in den Markt eingeführt. Fenoxycarb hemmt bei Insekten die Metamorphose zum adulten Stadium und stört Häutungen in frühen Larvenstadien. Die Wirkung dieser Substanz wurde für zahlreiche in der Landwirtschaft und im Vorratsschutz wichtige Insekten getestet. In ihren Untersuchungen mit Termiten hat Jones (1989) festgestellt, dass Fenoxycarb eine vielversprechende Substanz für Köderverfahren ist. 1995 stellte Valcke die Verbindung unter der Bezeichnung FAROX ${ }^{\mathrm{TM}}$ als Wirkstoff zur Kontrolle von holzzerstörenden Insekten vor. Einsatzmöglichkeiten von Insektenhormon-Analoga in Holzschutzmitteln wurden von Graf et al. (2002) umfangreich getestet.

Im Review-Verfahren zur EU-Biozidproduktenrichtlinie (Richtlinie 98/8/EC) wird zurzeit über die Aufnahme von Fenoxycarb als Wirkstoff zur Anwendung in Holzschutzmitteln in den Anhang I der Richtlinie entschieden.

In Deutschland gibt es Fenoxycarb-haltige Holzschutzmittel zur Vorbeugung gegenüber Insektenbefall mit einer Zulassung vom Deutschen Institut für Bautechnik (DIBt) seit 1998 und mit einem RAL-Gütezeichen seit 1999. Gegenwärtig sind wasserverdünnbare bzw. auch lösemittelbasierte Fenoxycarb-haltige Produkte zugelassen, die in Oberflächenverfahren und/oder Druckverfahren eingesetzt werden können (Stand Januar 2008). Die Auf- bzw. Einbringmengen für Fenoxycarb ins Holz sind wegen der hormonähnlichen Wirkung im Vergleich zu anderen Insektiziden sehr gering und betragen je nach Produkttyp 2 bis $20 \mathrm{mg} / \mathrm{m}^{2}$ bzw. etwa 0,5 bis $3 \mathrm{~g} / \mathrm{m}^{3}$. In Abhängigkeit vom Verfahren sind Konzentrationen von 1 bis ca. 20 mg je kg Holz zu erwarten.

Zur quantitativen Analyse muss Fenoxycarb durch ein geeignetes Verfahren aus behandeltem Holz extrahiert werden. Fenoxycarb ist in Wasser wenig $\left(6 \mathrm{mg} / \mathrm{l}\right.$ bei $\left.25^{\circ} \mathrm{C}\right)$, in vielen organischen Lösungsmitteln dagegen sehr gut löslich (z.B. > $500 \mathrm{~g} / \mathrm{l}$ in Ethanol und Methanol bei $25^{\circ} \mathrm{C}$ ). Zur Extraktion von Fenoxycarb aus fein gemahlenem Holz bietet sich die Verwendung von Methanol an. Zur Quantifizierung von Fenoxycarb aus Pflanzenschutzmitteln und Pflanzenextrakten empfehlen Haenni und Mueller (1988) Hochdruckflüssigchromatographie (HPLC) an RP18-Säulen in Verbindung mit UV-Detektion. Daneben gilt Gaschromatographie (GC) z.B. in Verbindung mit Massenspektrometrie (MS) als grundsätzlich geeignet zur Analyse von Fenoxycarb. 
Verfahrensvorschläge zur Analyse von Holzschutzmitteln aus behandeltem Holz werden von den Mitgliedern des UA 6.2 „Analytik“ der DGfH (Unterausschuss der Deutschen Gesellschaft für Holzforschung) auf Praxistauglichkeit getestet und auf der DGfH-Website zur Verfügung gestellt. Die geprüften Methoden sollen als Anregung für Anwender dienen, die entsprechende Verfahren z.B. für die Qualitätskontrolle von behandeltem Holz etablieren wollen. Im Folgenden soll über Erfahrungen aus Ringversuchen mit HPLC-Verfahren zur Analyse von Fenoxycarb und einige vergleichende GC/MS-Analysen berichtet werden.

\section{Material und Methoden \\ 2.1 Material}

Sowohl dotierte Holzproben als auch Holzextrakte mit definiertem Fenoxycarb-Gehalt wurden von einem Teilnehmer für Ringversuche in 10 weiteren beteiligten Laboratorien zur Verfügung gestellt. Die Versuche umfassten 3 Serien.

1. Serie: Sägemehl von Kiefernsplintholz (Pinus sylvestris) wurde mit in Methanol gelöstem Fenoxycarb versetzt (Sollgehalt: $10 \mathrm{mg} / \mathrm{kg}$ lufttrockenes Holz).

2. Serie: Undotierte und mit Fenoxycarb behandelte Prüfkörper aus Kiefernsplintholz wurden mit Methanol extrahiert und diese Extrakte mit unterschiedlichen Fenoxycarb-Mengen versetzt (Sollgehalte: 0,5 bis $2,5 \mu \mathrm{g} / \mathrm{ml}$, entsprechen Gehalten von 10 bis $50 \mathrm{mg} / \mathrm{kg}$ Fenoxycarb im Holz).

3. Serie: Prüfkörper aus Kiefernsplintholz wurden mit in Methanol gelöstem Fenoxycarb bzw. mit einem Fenoxycarb-haltigen Holzschutzmittel behandelt (Sollgehalte zwischen 10 und $20 \mathrm{mg} / \mathrm{kg}$ lufttrockenes Holz), anschließend gemahlen und gesiebt (Maschenweite $720 \mu \mathrm{m}$ ). Außerdem wurde beim Hersteller des Versuchsmaterials eine Holzprobe extrahiert und in Teilproben an die übrigen Teilnehmer versandt.

Zu jeder Versuchsserie wurde Probenmaterial von unbehandeltem Kiefernholz zur Verfügung gestellt.

\subsection{Extraktionsverfahren}

1 bis $2 \mathrm{~g}$ des Holzmehls wurde in ein verschließbares Glasgefäß eingewogen. Das zwanzigfache Volumen an Methanol bezogen auf die Holzeinwaage (z.B.: $20 \mathrm{ml}$ Methanol auf $1 \mathrm{~g} \mathrm{Holz}$ ) wurde zugegeben und die erhaltene Suspension unter gelegentlichem Aufschütteln für $2 \mathrm{~h}$ im Ultraschallbad extrahiert bzw. alternativ für $2 \mathrm{~h}$ gerührt (Magnetrührer) oder geschüttelt (Schüttelmaschine, Überkopfschüttler). Nach Absetzen der Holzpartikel wurde ein aliquoter Teil der überstehenden Extraktionslösung über einen 0,45 $\mu \mathrm{m}$ PTFE-Membranfilter filtriert und zur Analyse verwendet.

Hinweis: Es wird empfohlen, die ersten 1 bis 2 Milliliter des Filtrats zu verwerfen.

\subsection{HPLC-Methode}

Die Extrakte wurden an RP18-Phasen mittels eines Elutionsgradienten aus 0,15 \% w/v Ammoniumacetat in Wasser und Acetonitril aufgetrennt und durch UV-Detektion bei $230 \mathrm{~nm}$ quantitativ analysiert. Als geeignet haben sich die folgenden Säulen erwiesen: Xterra ${ }^{\circledR}$ MS C18 
(5 $\mu \mathrm{m}, 4,6^{\star} 250 \mathrm{~mm}$ ), Phenomenex Luna C18(2) ( $3 \mu \mathrm{m}, 4,6^{\star} 150 \mathrm{~mm}$ ), Gemini NX (3 $\mu \mathrm{m}$, 4,6*150 mm), Waters $x$-Bridge C18 (3,5 $\left.\mu \mathrm{m}, 4,6^{*} 100 \mathrm{~mm}\right)$. Je nach Säule wurden Flussraten zwischen 1 und $2 \mathrm{ml} / \mathrm{min}$ eingestellt und der Gradient angepasst (siehe Tabelle 1). Das Injektionsvolumen betrug 10 bis $15 \mu$, die Analysendauer lag bei 15 bis $20 \mathrm{~min}$. Die Analysen erfolgten bei $30{ }^{\circ} \mathrm{C}$ bzw. Raumtemperatur. Mit den in Tabelle 1 dargestellten Gradienten liegt die Retentionszeit für Fenoxycarb bei 10 bis 11 min. Zur Kalibrierung wurden FenoxycarbStandards mit Gehalten von 0,5 bis $5 \mathrm{ng} / \mu \mathrm{l}$ bzw. 0,25 bis $2 \mathrm{ng} / \mu \mathrm{l}$ verwendet.

\subsection{GC/MS-Analysen}

GC/MS-Analysen erfolgten nach den jeweiligen Prüfmethoden der Teilnehmer, z.B. an ZB-5ms, mit Helium als Trägergas, Injektion von $1 \mu \mathrm{lim}$ Split-Modus (5:1) bei $260{ }^{\circ} \mathrm{C}$ und einem Ofentemperaturprogramm, das bei $80{ }^{\circ} \mathrm{C}$ startet, mit $15{ }^{\circ} \mathrm{C} / \mathrm{min}$ auf $260{ }^{\circ} \mathrm{C}$ aufheizt und diese Temperatur für 4 min hält. Das Fenoxycarb-Signal erscheint bei ca. 12,5 min und wird über das Massensignal $\mathrm{m} / \mathrm{z} 116$ ausgewertet.

\section{$3 \quad$ Ergebnisse}

Abbildung 2 zeigt beispielhaft ein HPLC-Chromatogramm eines Holzextraktes. Bei direkter Analyse von Holzextrakten ist das Signal für Fenoxycarb im Vergleich zu den übrigen Komponenten der Extrakte sehr klein. Der vergrößerte Ausschnitt des Fenoxycarb-Peaks macht deutlich, dass eine weitere aus dem Holz stammende unbekannte Komponente aus dem Gemisch durch das gewählte chromatographische Verfahren nicht vollständig abgetrennt wurde. Diese zusätzliche Komponente wurde auch im Extrakt einer unbehandelten Holzprobe beobachtet (nicht dargestellt). Auch mit anderen Trennmaterialien wurde keine vollständige Trennung von Fenoxycarb und der vermutlich aus dem Holz stammenden Komponente erreicht. Die Ergebnisse der einzelnen Laboratorien mit den Proben der ersten Versuchsserie sind in Abbildung 3 dargestellt. Der Mittelwert der HPLC-Analysen lag mit 9,8 mg/kg im Bereich des Sollwertes von $10 \mathrm{mg} / \mathrm{kg}$. Bei den GC/MS-Analysen wurden im Mittel nur 7,6 mg/kg gefunden. Die prozentuale Standardabweichung der Messwerte zwischen den Laboratorien lag bei den HPLC-Analysen bei $13 \%$, bei den GC/MS-Analysen bei $17 \%$. Es wurde keine Tendenz zu höheren Extraktionsausbeuten bei den verschiedenen angewandten Extraktionsverfahren beobachtet (nicht dargestellt). Ein direkter Vergleich der Extraktionsausbeute durch Rühren bzw. Rühren mit einer zusätzlichen Ultraschallbehandlung im Labor L6 ergab Analysenwerte von 9,9 bzw. 9,7 mg/kg. Lediglich im Labor L10 wurden durch Überkopf-Schütteln höhere Ausbeuten erreicht als mit dem Ultraschallbad (8,9 bzw. 7,4 mg/kg).

Die Möglichkeit, Fenoxycarb durch GC/MS zu analysieren, wurde in den nachfolgenden Versuchen nicht verfolgt.

Parallele HPLC-Analysen von mit Fenoxycarb aufgestockten Holzextrakten lieferten bei allen Teilnehmern Resultate im Bereich der Sollwerte (siehe Tabelle 2 und Abbildung 4). Eine statistische Auswertung der Versuchsdaten ergab für den Messbereich von 0,5 bis $2,5 \mu \mathrm{g} / \mathrm{ml}$ einen Korrelationskoeffizienten von 0,9995 zwischen Soll- und Analysenwerten und für die untersuchten Extrakte Standardabweichungen von 0,1 bis $0,3 \mu \mathrm{g} / \mathrm{ml}$ für die Analysenwerte der 
einzelnen Laboratorien. Das entspricht ca. 7 bis $17 \%$ der zu messenden Konzentrationen und liegt im Bereich der Streuung der ersten Versuchsserie, bei der Holzproben von den einzelnen Teilnehmern selbst extrahiert wurden.

Auch für die in einer dritten Versuchsserie untersuchten Holzproben und den bereitgestellten Holzextrakt lagen die Mittelwerte der Analysenwerte sowohl für den Holzextrakt als auch für die Holzproben im Bereich der Sollwerte (siehe Tabelle 3 und Abbildung 5). Die Standardabweichungen für die Mess-Serien, bei denen das Holz von den Teilnehmern extrahiert wurde, lagen mit 16 bzw. $18 \%$ nur wenig über der für den bereitgestellten Holzextrakt (13\%). Auffallend ist auch, dass die Resultate der einzelnen Laboratorien relativ zu den Messergebnissen der übrigen Laboratorien für die Analysen der Holzextrakte mit denen für die Holzproben übereinstimmen.

\section{Diskussion}

Die Vergleichsanalysen mit Fenoxycarb-behandeltem Holz und Holzextrakten belegen, dass es mit dem beschriebenen Verfahren möglich ist, Fenoxycarb aus behandeltem Holz in einem Konzentrationsbereich von 10 bis $20 \mathrm{mg} / \mathrm{kg}$ Holz zu quantifizieren. Durch Extraktion mit Methanol erreicht man gute Ausbeuten. Das zeigt sich einerseits darin, dass die Analysenergebnisse in allen Versuchsserien im Bereich der Sollwerte liegen (Abbildung 2 bis 5 , Tabellen 2 und 3). Bestätigt wird dies auch dadurch, dass die Analysenwerte für die Proben, die anteilig aus Holz extrahiertes Fenoxycarb enthielten, und für die Proben, denen Fenoxycarb ausschließlich nach der Extraktion zugegeben wurde, dieselbe lineare Abhängigkeit aufweisen (Abbildung 4). Die Extraktionsausbeuten durch Rühren und verschiedene Schüttelverfahren waren ähnlich hoch. Aus der geringen Anzahl der Parallelversuche mit den verschiedenen Extraktionsverfahren kann jedoch keine gesicherte Aussage abgeleitet werden. Allerdings muss man bei direkter Analyse der Extrakte mittels HPLC/UV mit relativ hohen Messunsicherheiten rechnen, die im Wesentlichen darauf beruhen, dass Fenoxycarb mit den derzeit angewandten Trennmethoden nicht vollständig von Inhaltsstoffen des Holzes getrennt wird (siehe Abbildung 2). Ähnliche Standardabweichungen der Analysenwerte für Holzproben und bereitgestellte Holzextrakte (Tabellen 2 und 3) weisen darauf hin, dass die Unterschiede zwischen den Ergebnissen der beteiligten Laboratorien offensichtlich eher auf den Analysenverfahren als auf der Probenextraktion beruhen.

Zur Kontrolle von Fenoxycarb-Gehalten in behandeltem Holz reicht die durch direkte HPLC/UV-Analyse von Holzextrakten erreichte Messempfindlichkeit nicht aus, da die Konzentrationen in behandeltem Holz je nach Schutzmittel und Behandlungsverfahren bei 1 bis $20 \mathrm{mg} / \mathrm{kg}$ liegen können. Um Fenoxycarb im gesamten Konzentrationsbereich quantifizieren zu können, sind weitere Methodenentwicklungen erforderlich. Ansatzpunkte dafür bestehen einerseits in einer selektiven Anreicherung des Wirkstoffs aus den Extrakten und andererseits in einer spezifischen Detektion, z.B. durch Massenspektrometer. Graf et al. (2002) beschreiben die Anreicherung von Fenoxycarb aus Eluaten von Auswaschexperimenten durch Festphasenextraktion an RP-Säulen. Dieses Verfahren ist auf Methanolextrakte allerdings nicht 
direkt übertragbar, da in Methanol gelöstes Fenoxycarb nur schlecht an die unpolare stationäre Phase bindet.

Allen Anwendern wird empfohlen, die Eignung des gewählten Verfahrens anhand von Holzproben mit bekanntem Fenoxycarb-Gehalt zu überprüfen. Zu beachten ist, dass in der Praxis die Extrahierbarkeit des Wirkstoffs und die Zusammensetzung der Extrakte auch von der Holzart und der jeweiligen Formulierung beeinflusst sein können. Deshalb können Erfahrungen mit Proben einer Holzart nicht ungeprüft auf praxisüblich behandeltes Holz einer anderen Holzart übertragen werden.

\section{$5 \quad$ Literatur}

Deutsches Institut für Bautechnik (2008) Holzschutzmittelverzeichnis. DIBt Schriften des Deutschen Instituts für Bautechnik. Erich Schmidt Verlag

Dorn S, Frischknecht ML, Martinez V, Zurflüh R, Fischer U (1981) A novel non-neurotoxic insecticide with a broad activity spectrum. Z. Pflanzenkrankh Pflanzenschutz 88(5) 169275

Graf E, Barkhoff M, Hamberg R, Büttner H, Pallaske M (2002) The use of insect hormones as non-neurotoxic insecticides in wood preservatives, IRG/WP 02-30277, 1-39

Haenni RP, Mueller PA (1988) Fenoxycarb in Analytical Methods for Pesticides and Plant Growth Regulators. Vol. XVI, Academic Press Inc. 21-29

Jones SC (1989) Field evaluation of fenoxycarb as a bait toxicant for subterranean termite control. Sociobiology 15(1) 33-41

Tomlin C (ed.) (1994) The Pesticides Manual - A World Compendium of Pesticides. The British Crop Protection Council, 10. Auflage

Valcke A (1995) FAROX ${ }^{\mathrm{TM}}$, a novel insect growth regulator for use against wood-boring insects, IRG/WP 95-30080, 1-5

\section{$6 \quad$ Internet-Links}

Website der Europäischen Kommission mit Informationen zur Umsetzung der Biozidproduktenrichtlinie. http://ec.europa.eu/environment/biocides/index.htm

Thieme Römpp Online, Version 3.2. http://www.roempp.com/prod/index1.html

Website der DGfH. http://www.dgfh.de/ 


\section{Bestimmung von Fenoxycarb in Holz mittels Hochdruckflüssigchromatographie}

Schoknecht $\mathrm{U}^{1}$, Bornkessel, $\mathrm{C}^{2}$, Fürhapper $\mathrm{Ch}^{3}$, Gunschera $\mathrm{J}^{4}$, Härtner $\mathrm{H}^{5}$, Hill $\mathrm{R}^{6}$, Kochan, J. ${ }^{7}$ Melcher $\mathrm{E}^{8}$, Wilken $\mathrm{U}^{9}$, Wittenzellner $\mathrm{J}^{10}$, Wobst $\mathrm{M}^{11}$

\section{Unterschriften zu den Abbildungen}

Abb. 1: Strukturformel von Fenoxycarb (CAS-Nr.: 72490-01-8)

Fig. 1: Chemical structure of fenoxycarb (CAS-No.: 72490-01-8)

Abb. 2: HPLC-Chromatogramm eines Methanol-Extraktes einer Holzprobe, der an einer Phenomenex-Säule vom Typ Luna C18(2) ( $3 \mu \mathrm{m}, 4,6^{*} 150 \mathrm{~mm}$ ) mit einem Gradienten aus $0,15 \%$ Ammoniumacetat und Acetonitril eluiert wurde (Gradient siehe Tabelle 1). Die Detektion erfolgte mit einem Diodenarraydetektor bei $230 \mathrm{~nm}$. Das Holz wurde vor dem Mahlen mit einem Holzschutzmittel behandelt und hatte einen Sollgehalt von 13,5 mg Fenoxycarb/kg lufttrockenes Holz. Die Fenoxycarb-Konzentration des Extraktes lag bei ca. $0,8 \mathrm{ng} / \mu \mathrm{l}$.

Fig. 2: HPLC chromatogram of a methanolic extract of wood eluted on a Phenomenex column Type Luna C18(2) ( $\left.3 \mu \mathrm{m}, 4.6^{*} 150 \mathrm{~mm}\right)$ with a gradient of $0.15 \%$ ammonium acetate and acetonitrile (see Table 1 for the gradient). Detection was performed by a diode array detector at $230 \mathrm{~nm}$. The wood was treated before grinding and had an expected concentration of $13.5 \mathrm{mg}$ fenoxycarb/kg air dried wood. The concentration of fenoxycarb in the extract was approx. $0.8 \mathrm{ng} / \mu \mathrm{l}$.

Abb. 3: Ergebnisse von Ringversuchen für Fenoxycarb aus Kiefernholzproben mittels HPLC/UV und GC/MS. Der Sollgehalt der Holzproben lag bei $10 \mathrm{mg}$ Fenoxycarb/kg lufttrockenes Holz. Die starken Linien zeigen den Mittelwert für das jeweilige Analysenverfahren, die schwächeren Linien den Bereich zwischen Mittelwert +/- zweifacher Standardabweichung. Die Dreiecke zeigen das höchste bzw. niedrigste Resultat für parallele Probenextraktionen. Die beteiligten Laboratorien sind mit L1 bis L11 bezeichnet. Im Labor L6 erfolgten Extraktionen sowohl durch Rühren (L6-1) als auch Rühren und Ultraschallbehandlung (L6-2), im Labor 10 durch Ultraschall (L10-1) bzw. Überkopfschüttler (L10-2).

Fig. 3: Results of comparative analyses of fenoxycarb in pine wood by HPLC/UV and GC/MS. The expected concentration of fenoxycarb in the wood samples was $10 \mathrm{mg} / \mathrm{kg}$ air dried wood. The solid lines represent mean results of each analytical method, the weaker lines indicate the range within twofold standard deviation. The triangles indicate maximum and minimum results for parallel extractions, respectively. The laboratories involved are indicated as $L 1$ to $L 11$. The 
extractions were performed by both stirring (L6-1) and stirring combined with ultrasonication (L6-2) in laboratory L6, and by both ultrasonication (L10-1) and overhead shaking (L10-2) in laboratory L10.

Abb. 4: Ergebnisse von HPLC/UV-Analysen für Teilproben aus Holzextrakten, denen Fenoxycarb zugesetzt wurde. Gezeigt sind Mittelwerte mit Standardabweichungen für die Messergebnisse der beteiligten Laboratorien ( $n=6$ bzw. 7, siehe Tabelle 2). Der niedrigste Messwert für Extrakte von behandeltem Holz entspricht der Fenoxycarb-Konzentration im Holz ohne weiteren Zusatz des Wirkstoffs zum Extrakt.

Fig. 4: Results of HPLC/UV analyses of subsamples of wood extracts with an additional dosage of fenoxycarb. The mean values of the results of the involved laboratories $(n=6$ and 7 , respectively, see Table 2) are demonstrated with standard deviations. The lowest value for the extracts from treated timber corresponds to the concentration of fenoxycarb in wood without further addition of the active ingredient to the extract.

Abb. 5: Ergebnisse von Vergleichsanalysen für Fenoxycarb in einem bereitgestellten Holzextrakt und gemahlenen Holzproben mittels HPLC/UV. Die starken Linien zeigen den Mittelwert für die jeweilige Probe, die schwächeren Linien den Bereich zwischen Mittelwert +/zweifacher Standardabweichung. Die Dreiecke zeigen das höchste bzw. niedrigste Resultat für parallele Probenextraktionen. Die beteiligten Laboratorien sind mit L1 bis L6 bezeichnet; $n$ bezeichnet die Anzahl der in dem jeweiligen Labor parallel durchgeführten Extraktionen. Die Ergebnisse für die Holzproben sind auf lufttrockenes Holz bezogen.

Fig. 5: Results of comparative analyses of fenoxycarb in a provided wood extract and ground wood samples by HPLC/UV. The solid lines represent mean results of each analytical method, the weaker lines indicate the range within twofold standard deviation. The triangles indicate maximum and minimum results for parallel extractions, respectively. The laboratories involved are indicated as $L 1$ to $L 6 ; n$ is the number of parallel experiments that were performed in each laboratory. The results are related to air dried wood. 
Tabelle 1: Beispiele für Elutionsgradienten zur HPLC-Analyse von Fenoxycarb in Holzextrakten (Flussrate jeweils $1,5 \mathrm{ml} / \mathrm{min}$ )

Table 1: Examples of elution gradients for HPLC analysis of fenoxycarb in wood extract (flow rate $1.5 \mathrm{ml} / \mathrm{min}$ in each case)

\begin{tabular}{|c|c|c|c|}
\hline \multicolumn{2}{|c|}{ Xterra ${ }^{\circledR}$ MS $250 \mathrm{~mm}$} & \multicolumn{2}{c|}{ Phenomenex Luna C18(2) } \\
\hline Zeit [min] & $\%$ Acetonitril & Zeit [min] & \% Acetonitril \\
\hline 0 & 50 & 0 & 50 \\
\hline 10 & 50 & 10 & 50 \\
\hline 10,1 & 100 & 10,1 & 95 \\
\hline 15 & 100 & 15 & 95 \\
\hline 15,1 & 50 & 15,1 & 50 \\
\hline 20 & 50 & 18 bzw. 20 & 50 \\
\hline
\end{tabular}


Tabelle 2: Ergebnisse aus vergleichenden Analysen von Fenoxycarb in Holzextrakten

Table 2: Results of comparative analyses of fenoxycarb in wood extracts

\begin{tabular}{|c|c|c|c|c|c|}
\hline \multirow{2}{*}{ Fenoxycarb im Holz } & \multicolumn{5}{|c|}{ Fenoxycarb-Konzentration in aufgestockten Holzextrakten } \\
\cline { 6 - 6 } & Sollwerte & Analysenwert & \multirow{2}{*}{$\mathrm{n}^{* *}$} & \multicolumn{2}{|c|}{ Standardabweichung } \\
\cline { 5 - 6 } $\mathrm{mg} / \mathrm{kg}$ & $\mu \mathrm{g} / \mathrm{ml}$ & $\mu \mathrm{g} / \mathrm{ml}$ & & $\mu \mathrm{g} / \mathrm{ml}$ & $\%$ \\
\hline \hline 0 & 0,50 & 0,54 & 7 & 0,09 & 17 \\
\hline ca. 12 & 0,60 & 0,61 & 6 & 0,09 & 14 \\
\hline ca. 12 & 1,00 & 1,01 & 6 & 0,08 & 8 \\
\hline ca. 12 & 1,40 & 1,41 & 6 & 0,09 & 7 \\
\hline ca. 12 & 2,20 & 2,21 & 6 & 0,15 & 7 \\
\hline 0 & 2,50 & 2,59 & 7 & 0,27 & 10 \\
\hline
\end{tabular}

${ }^{*}$ Mittelwert der Analysenergebnisse aus $\mathrm{n}$ Laboratorien, ${ }^{* *}$ Anzahl der beteiligten Laboratorien 
Tabelle 3: Ergebnisse aus vergleichenden Analysen von Fenoxycarb in einem bereitgestellten Holzextrakt und Holzmehl aus behandeltem Kiefernholz

Table 3: Results of comparative analyses of Fenoxycarb in a provided wood extract and sawdust of treated pine wood

\begin{tabular}{|c|c|c|c|c|c|}
\hline \multirow{2}{*}{ Probe } & \multicolumn{5}{|c|}{ Fenoxycarb-Konzentration } \\
\cline { 2 - 6 } & Sollwerte & Analysenwert & $\mathrm{n}^{* *}$ & \multicolumn{2}{|c|}{ Standardabweichung } \\
\cline { 2 - 6 } & $\mu \mathrm{g} / \mathrm{ml}$ & $\mu \mathrm{g} / \mathrm{ml}$ & & $\mu \mathrm{g} / \mathrm{ml}$ & $\%$ \\
\hline \hline Holzextrakt & 1,04 & 1,00 & 6 & 0,130 & 13 \\
\hline \hline $\begin{array}{c}\text { Holzprobe mit Fenoxycarb } \\
\text { in Methanol }\end{array}$ & $\mathrm{mg} / \mathrm{kg}$ & $\mathrm{mg} / \mathrm{kg}$ & & $\mathrm{mg} / \mathrm{kg}$ & $\%$ \\
\hline $\begin{array}{c}\text { Holzprobe mit Fenoxycarb } \\
\text { in Holzschutzmitte| }^{* * *}\end{array}$ & 19,4 & 19,8 & 5 & 3,5 & 18 \\
\hline
\end{tabular}

${ }^{*}$ Mittelwert der Analysenergebnisse aus $\mathrm{n}$ Laboratorien, ${ }^{* *}$ Anzahl der beteiligten Laboratorien,

*** Daten sind auf lufttrockenes Holz bezogen 


\section{Abbildung 1}<smiles>CCOC(=O)NCCOc1ccc(Oc2ccccc2)cc1</smiles> 
Abbildung 2

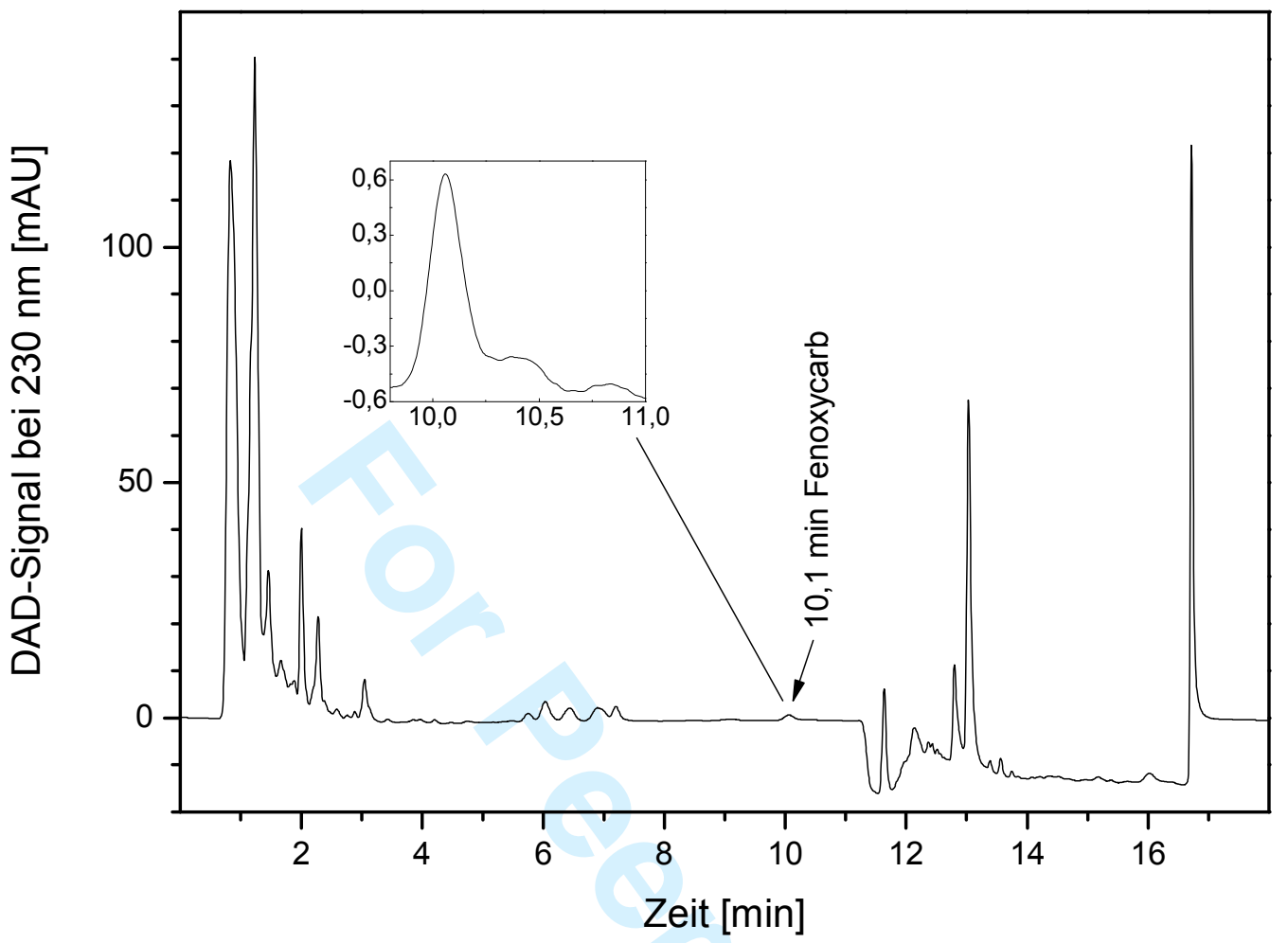


Abbildung 3

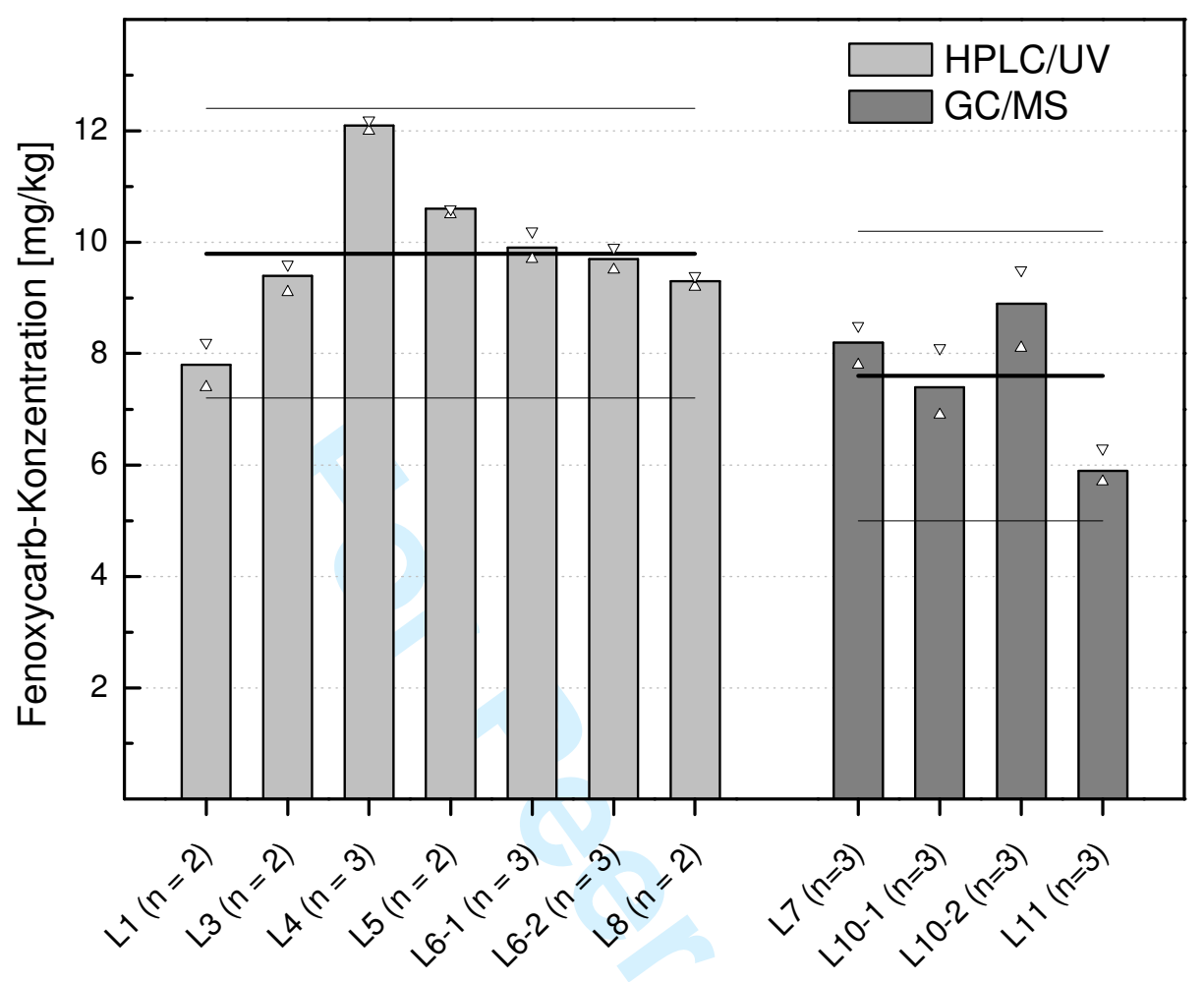


Abbildung 4

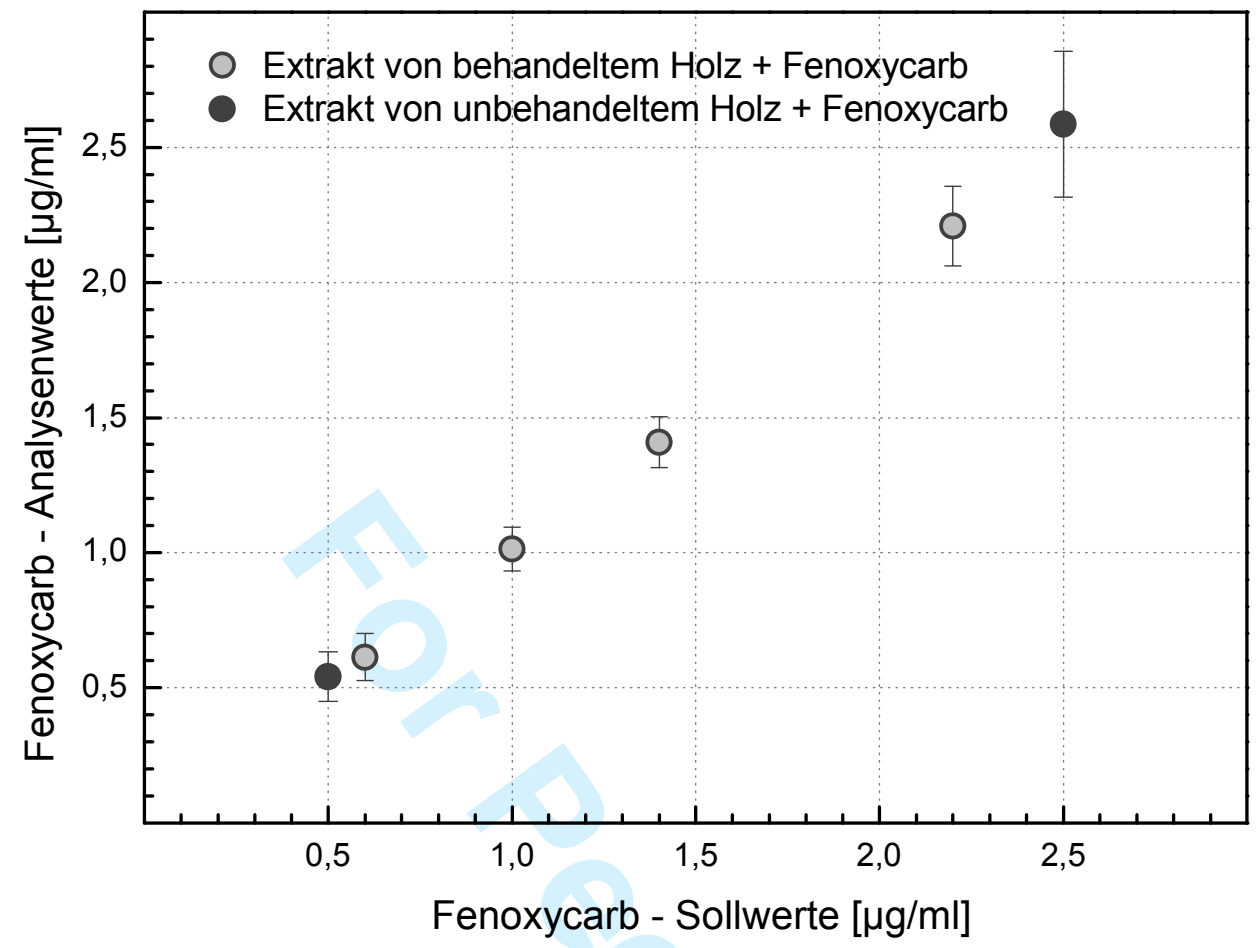


Abbildung 5
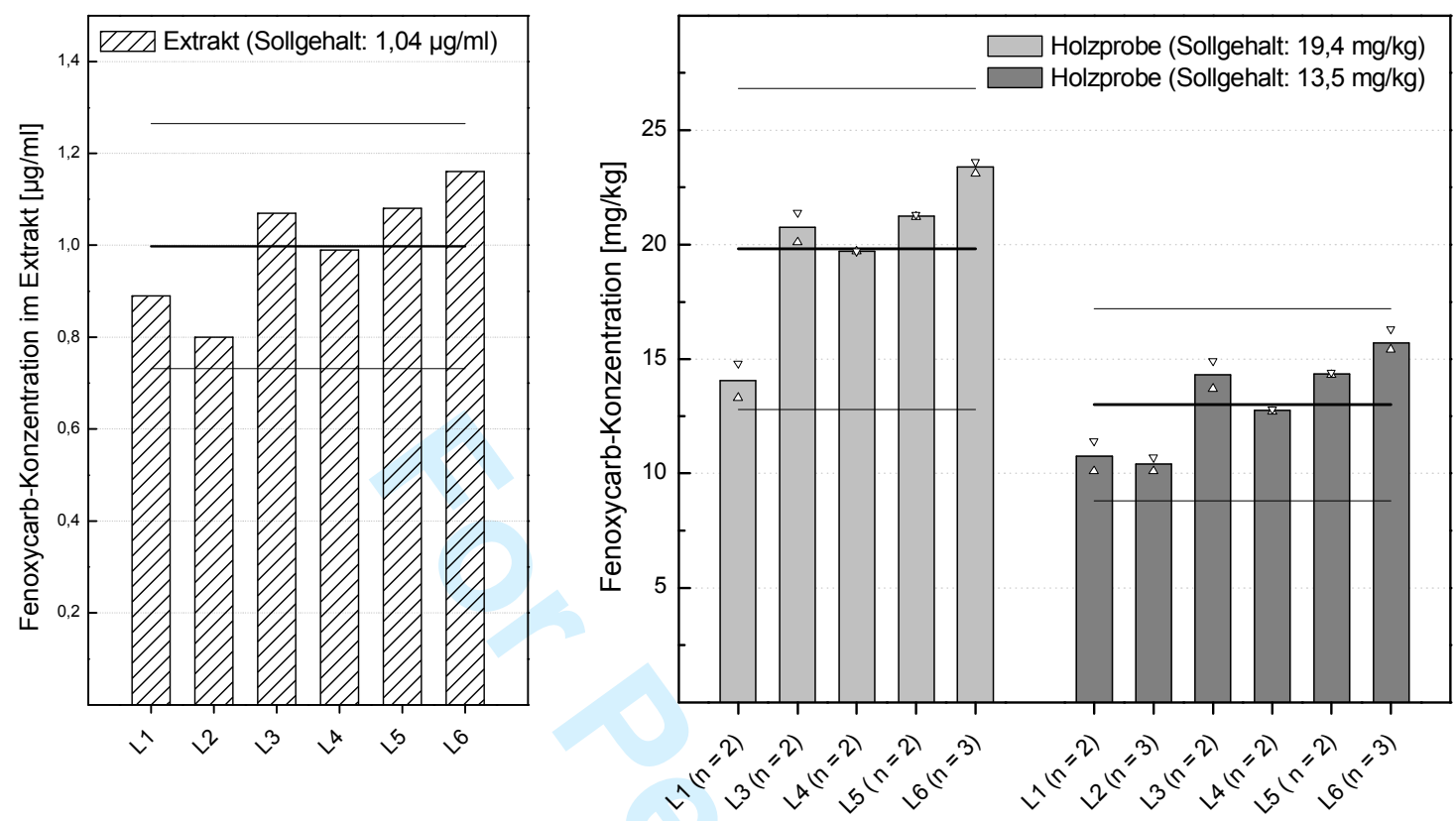

29

30

31

32

33

34

35

36

37

38

39

40

41

42

43 